\title{
Occlusal Interferences: How Can This Concept Influence The Clinical Practice?
}

\author{
Adriano Fonseca Limaa \\ Andrea Nóbrega Cavalcantia \\ Luis Roberto Marcondes Martins ${ }^{a}$ \\ Giselle Maria Marchia
}

\section{ABSTRACT}

This brief review discusses the role of occlusal interferences on the development and progression of temporomandibular dysfunction (TMD) and postural disorders. The eligible literature is described and critically presented to demonstrate that occlusal interferences are an important factor in the risk of TMD. Several studies have demonstrated through their findings that the use of occlusal adjustments, whether or not associated with restorative procedures, might avoid the development of articular problems in vulnerable patients. The occlusal interferences caused by restorative procedures or orthodontic treatment can cause TMDs in susceptible patients, and occlusal adjustments can be an alternative in the treatment of these TMDs. (Eur J Dent 2010;4:487-491)

Key words: Temporomandibular dysfunction; Occlusal interferences.

\section{INTRODUCTION}

Occlusal adjustment can be defined as the removal of occlusal interferences, through selective tooth grinding or through the use of restorative materials. The aim of such an intervention is to obtain a stable occlusal relationship, with no premature contacts or mandibular excursion.

a Department of Restorative Dentistry, Piracicaba School of Dentistry, State University of Campinas-UNICAMP, Piracicaba, SP, Brazil.

Corresponding author: Adriano Fonseca Lima Departamento de Odontologia Restauradora Faculdade de Odontologia de Piracicaba - UNICAMP Av: Limeira - Areião. CEP: 13414-903, Piracicaba, SP, Brazil.

Phone: +55-19-2106-5340 Fax: +55-19-2106-5218

E-mail: lima.adfagmail.com
The adjustments can be performed in different situations, such as 1) after performing direct or indirect restorations, in order to establish a better relationship with the antagonist teeth, or 2) in the presence of non-uniform tooth wear, which might result in premature contacts and deviations on excursive mandibular movements.

Several studies have evaluated the role of occlusal interferences on the etiology of temporomandibular joint disorders. ${ }^{1-10}$ Nevertheless, there is no consensus on the importance of such a theme for the development of TMJ dysfunctions. The greater limitation on obtaining an appropriate explanation for the role of occlusal adjustment on the etiology of TMDs is the number of variables associated with such dysfunction, which renders 
difficult the studies' standardization and the consequent discussion of their results. Also, physiologic factors, traumas, articular disc dislodgment, condylar position, and pathologies can lead to TMDs. ${ }^{11,12}$

Given the elevated controversy and extreme importance of such a theme for clinical practice, it might be helpful for correct clinical management to review the information on the influence of occlusal interferences on the etiology of TMDs, increasing the possibility of success in the treatment of this highly prevalent dysfunction.

\section{OCCLUSAL ADJUSTMENT AND ITS RE-} LATIONSHIP TO TEMPOROMANDIBULAR DISORDERS AND CHRONIC PAIN

Studies have tried to establish the relationship between occlusal interferences and TMDs. Kopp ${ }^{13}$ analyzed the effect of professional advisement during occlusal therapy. During the first appointment for this study, patients had received instructions only about their habits and about the possible causes of TMDs. In a second appointment, one group of patients was submitted to occlusal therapy, and another group remained untreated, but received different instructions about their habits. The results showed that both situations ladvisement and occlusal therapyl resulted in decreased symptoms.

In spite of its interesting findings, this study presented some limitations; for example, the occlusal interventions consisted of several modalities of treatment, such as occlusal adjustment, installation of occlusal splints, and even complete dentures. Because of these factors, it might be reasonable to assume that such results cannot be directly extrapolated to the clinical management of patients with TMDs.

In 1986, Forssell ${ }^{14}$ evaluated the efficiency of occlusal adjustments in reducing or eliminating TMJ dysfunctions. Forty-eighty patients were treated with occlusal adjustments, of which 19 used occlusal splints. An additional 43 patients received a false occlusal adjustment, consisting of a placebo group. Symptoms associated with TMDs were investigated, and a neurologic examination of headaches was performed. The results demonstrated the reduction in clinical symptoms in both groups, although it was significantly greater for treated patients than for the ones from the pla- cebo group. Thus, this study was able to effectively demonstrate the efficiency of occlusal adjustment in the remission of symptoms related to TMDs. The statistical analysis demonstrated that this reduction in symptoms happened irrespective of the use of occlusal splints, reaffirming the effectiveness of the occlusal adjustment. These results were confirmed in another similar study, ${ }^{15}$ reinforcing the effectiveness of the occlusal adjustments.

The effectiveness of the occlusal adjustment and the use of occlusal splints, whether or not associated with occlusal interventions, were evaluated by Wenneberg et al. ${ }^{16}$ Patients were randomly divided into two treatment groups and were examined after two months by means of questionnaires, visual analogical scales (VAS), and clinical exams. The questionnaires indicated clinical improvement in the patients treated with both therapies, but the findings of the clinical exam were statistically significant only for patients from the mixed techniques group. The authors concluded that the use of occlusal splints in combination with occlusal adjustment is more effective for the treatment of TMDs than occlusal adjustments alone.

TMDs are not restricted to headaches in the region of the temporal or masseter muscles. The symptoms can be extended to the neck or to the superior region of the back. Karppinen et $\mathrm{al}^{8}$ investigated the importance of occlusal adjustment for the treatment of chronic neck- and headaches. Forty patients were selected after the observation of some criteria: need for neck and/or headache treatment, absence of clinical signs of TMD, nonexistence of removable prosthesis, and presence of posterior teeth. Patients were randomly allocated into two groups $(n=20)$; one group received occlusal adjustment, and the other received a false adjustment. Patients were analyzed after 6 weeks, 12 weeks and 60 months, using a visual analogical scale (VAS), a physical exam performed by a physiotherapist, an electromyography, and radiographies; however, the last two were not performed during the 60-month exam. The final analysis demonstrated that the occlusal adjustments were efficient in $80 \%$ of the cases after 60 months.

The physiotherapists observed a positive response in the occlusal adjustments as compared to the false adjustment, after comparing aches during head and neck movements. The authors have concluded that occlusal adjustment can be 
effective for the treatment of head and neck chronic pains.

Le Bell et $\mathrm{al}^{3}$ performed a study in which patients presenting TMDs (21 patients) and those who did not (26 patients) were distributed into four groups that received proper adjustments or simulations. During a two-week period, patients were instructed to report the intensity of nine different symptoms daily, using the VAS. The symptoms were occlusal discomfort, chewing difficulties, sensitivity of teeth, fatigue of the jaw, headache, facial pain, opening difficulty, bruxism, and ear symptoms.

The results were statistically associated with the presence or absence of TMJ disorders and to the implementation of occlusal adjustments. Patients with a history of TMDs, as well as the ones that received occlusal interventions, presented greater symptoms than the other groups. The most evident symptoms were occlusal discomfort and chewing difficulties, and the interaction between them was statistically significant. Patients with no history of TMDs who had received occlusal adjustments demonstrated symptoms similar to those of patients with a history of TMDs; nevertheless, these symptoms decreased until the final observation period. The authors have concluded that the different answers from patients with and without a history of TMDs may suggest dissimilar vulnerability to occlusal interferences, indicating the need for a reassessment of the etiology of TMDs.

The TMDs are a multidisciplinary and multifactorial dysfunction, since factors like occlusal balance, vertical dimension, muscle function, and psychological aspects are involved. Niemi et $\mathrm{al}^{4}$ evaluated the association of psychological factors and occlusal interferences. Twenty-six healthy women without a history of TMDs and 21 women with a history were evaluated. All women complaining of TMDs had been previously and successfully treated with routine therapies. Each group of patients was divided into two groups (performance of occlusal adjustments or placebo), and evaluated before the occlusal adjustments with a questionnaire. After the intervention, patients were examined for the level of psychological and somatic stress, coping strategies, personality characteristics, and health beliefs. The authors concluded that health, health hardiness, a positive socialization history, social support-seeking, and inhibition of aggression are associated with better adaptation to occlusal interferences; and patients with a history of TMDs presented more prominent results compared to the other group.

\section{RELATIONSHIP BETWEEN OCCLUSAL ADJUSTMENT AND POSTURAL STABILITY}

Occlusal interferences not only can affect the development and severity of TMDs, but also can influence the body posture and equilibrium. This postural instability can lead to damage, especially in patients who need the equilibrium to perform their actions adequately. ${ }^{17}$

Gangloff et al ${ }^{17}$ evaluated the influence of occlusion on the postural control and gaze stabilization of professional shooters. Thirty-six patients were selected; nevertheless, in the 6 months before the study, not all patients presented the muscular, articular, and osseous pains that could interfere with postural control. Patients with meniscus subluxation or luxation of the temporo mandibular joint were excluded from the study. Eighteen volunteers with great oral hygiene were included in the control group for postural evaluation. The test group was completed by the high-level shooters, who were investigated for gaze stabilization and postural control. For the evaluation, four mandibular positions were used: first, intercuspal occlusion (IO), defined as the interdigitation of all teeth without any type of manipulation; this is a neutral position, but not a symmetric one. The other three positions were tested by means of acrylic devices: centric relation (CR) and left and right laterality.

The postural evaluation through posturographic measurements (QFP SysteÁmes, Nice, France) were recorded in the same place and under the same environmental conditions for all control group subjects, and the results were statistically analyzed. For each occlusal relation, the test was conducted with opened and blinded eyes. In the professional shooters' group, four series of five shots (shooting distance: $10 \mathrm{~m}$ ) were performed in each of the four predefinite mandibular positions.

The best results were obtained in RC, followed by 10 , then lateral occlusion. The shooters presented better results in accordance with the best mandibular position. The authors have suggested that the mandibular position can be important to a high level sportsman who requires optimum balance control and posture for a better performance. 
The effect of different mandibular positions on postural stability was also evaluated by Bracco et al. ${ }^{18}$ Ninety-five subjects, with no signs and symptoms of temporomandibular disorder, were evaluated. None of the patients was informed about the aims of the study. The posture of patients was analyzed in three different occlusal positions: maximum intercuspidation; rest position, which is the habitual postural position of the mandible when at rest, with the condyles in a neutral, unstrained position in the glenoid fossa; and myocentric position, which is most commonly between 1 and $2 \mathrm{~mm}$ of vertical closure from the physiologic rest position that occurs when postural and masticatory muscles are simultaneously at their resting length and in balanced tonus with respect to one another and to a selected terminal contact therapeutic position. The patients' posture was analyzed by a computerized posturographic and stabilometric footboard. Data have shown a strong relation between mandibular position and body posture. The authors concluded that a good relation between chewing muscles and head and neck seems to be an important factor for postural stability.

Cuccia and Caradonna ${ }^{19}$ performed a review in which they searched such databases as Medline, PubMed, Embase, Web of Sciences, Cochrane Library, Cinahl, and Health Star for information on issues like body posture, temporomandibular disorders, and chewing muscles. The authors analyzed these and other issues, making correlations with the findings and demonstrating the importance of the theme and the need for further studies in this area. They have suggested that an interdisciplinary approach to TMDs, involving diverse specialists like dentists, physiotherapists, orthopedists, and psychologists, is fundamental for a broad, reliable, and successful treatment of the subject.

\section{DISCUSSION}

TMDs are pathologic conditions involving the masticatory muscles and head and neck postural muscles or, at the least, a combined pathologic condition of the muscles and temporo-mandibular joints. ${ }^{18}$ Considering this definition, the treatment of TMJ disorders by addressing the condylar position in relation to the glenoid fossa or to the chewing muscles like an individual approach, have a great chance of being unsuccessful, but may be necessary in some situations.
In accordance with the literature, occlusal interferences can play an important role in the development of TMDs. 3,4,20,21 These interferences can be formed by uneven tooth wear, but also by restorative procedures performed incorrectly, which can leads to a disharmonic relation between the arches. Therefore, after conducting direct or indirect restorations, a careful analysis of occlusal contacts should be performed, in order to avoid the creation of iatrogenic interferences that can produce the signs and symptoms of TMDs and postural disorders.

An occlusal interference can cause the alteration of muscular tonus, which can lead to pains in chewing and in the head and neck muscles. Patients with no history of TMDs are less vulnerable to occlusal alterations; ${ }^{3}$ nevertheless, in vulnerable patients, the symptoms can be exacerbated if the occlusal adjustment of rehabilitations is wrongly performed, compromising the harmonic relation between the arches. If the trigeminal afferences and proprioception are altered, these disorders can generate, in descending action, into an imbalance of postural muscles chains, finally causing postural modifications. ${ }^{18}$

From the information gathered, it seems to be evident that occlusal interferences can lead to the development of or to an increase in the severity of TMDs. Since occlusal therapy could induce a re-equilibrium of masticatory muscles, this reequilibrium could influence, in descending action, the whole body's postural muscles, resulting in an improved posture. ${ }^{18}$ Also, the relation between the upper and inner arches and the temporomandibular joint can generate alterations in the gaze stabilization and in the body posture, which indicate that the relevance of the occlusal adjustment on the treatment of TMDs should be reevaluated.

The authors should clarify that not only occlusal adjustments can be considered in TMD treatment, but also the use of occlusal splints, psychological therapy, and physiotherapist treatment. Each case should be considered singly and analyzed according the clinical situation.

As a further consideration, adjustments should be conducted only after the influence of interferences on the disorder has been confirmed through evaluating the signs and symptoms, and never in a prophylactic manner. ${ }^{1}$ Finally, a multidisciplinary approach should be employed in order to provide 
the most correct treatment and to achieve a greater likelihood of success.

\section{REFERENCES}

1. Barbosa G, Badaró Filho C, Fonseca R, Soares C, Neves F, Neto A. The role of occlusion and occlusal adjustment on temporomandibular dysfunction. Braz J Oral Sci 2004;3:589-594.

2. Le Bell Y, Jamsa T, Korri S, Niemi PM, Alanen P. Effect of artificial occlusal interferences depends on previous experience of temporomandibular disorders. Acta Odontol Scand 2002;60:219-222.

3. Le Bell Y, Niemi PM, Jamsa T, Kylmala M, Alanen P. Subjective reactions to intervention with artificial interferences in subjects with and without a history of temporomandibular disorders. Acta Odontol Scand 2006;64:59-63.

4. Niemi PM, Le Bell Y, Kylmala M, Jamsa T, Alanen P. Psychological factors and responses to artificial interferences in subjects with and without a history of temporomandibular disorders. Acta Odontol Scand 2006;64:300-305.

5. Tsukiyama Y, Baba K, Clark GT. An evidence-based assessment of occlusal adjustment as a treatment for temporomandibular disorders. J Prosthet Dent 2001;86:57-66.

6. Kuttila M, Le Bell Y, Alanen P. The concepts prevalence, need for treatment, and prevention of temporomandibular disorders: a suggestion for terminology. Acta Odontol Scand 1996;54:332-336.

7. Kuttila M, Le Bell Y, Savolainen-Niemi E, Kuttila S, Alanen $P$. Efficiency of occlusal appliance therapy in secondary otalgia and temporomandibular disorders. Acta Odontol Scand 2002;60:248-254.

8. Karppinen K, Eklund S, Suoninen E, Eskelin M, Kirveskari $P$. Adjustment of dental occlusion in treatment of chronic cervicobrachial pain and headache. J Oral Rehabil 1999;26:715-721.

9. Kirveskari P, Alanen P. Occlusal variables are only moderately useful in the diagnosis of temporomandibular disorder. J Prosthet Dent 2000;84:114-115.

10. Kirveskari P, Jamsa T, Alanen P. Occlusal adjustment and the incidence of demand for temporomandibular disorder treatment. J Prosthet Dent 1998;79:433-438.

11. Ash MM. Paradigmatic shifts in occlusion and temporomandibular disorders. J Oral Rehabil 2001;28:1-13.

12. Christensen GJ. Now is the time to observe and treat dental occlusion. J Am Dent Assoc 2001;132:100-102.

13. Kopp S. Short term evaluation of counselling and occlusal adjustment in patients with mandibular dysfunction involving the temporomandibular joint. JOral Rehabil 1979;6:101109.
14. Forssell H, Kirveskari P, Kangasniemi P. Effect of occlusal adjustment on mandibular dysfunction. A double-blind study. Acta Odontol Scand 1986;44:63-69.

15. Forssell H, Kirveskari P, Kangasniemi P. Response to occlusal treatment in headache patients previously treated by mock occlusal adjustment. Acta Odontol Scand 1987;45:7780.

16. Wenneberg B, Nystrom T, Carlsson GE. Occlusal equilibration and other stomatognathic treatment in patients with mandibular dysfunction and headache. J Prosthet Dent 1988;59:478-483.

17. Gangloff P, Louis JP, Perrin PP. Dental occlusion modifies gaze and posture stabilization in human subjects. Neurosci Lett 2000;293:203-206.

18. Bracco P, Deregibus A, Piscetta R. Effects of different jaw relations on postural stability in human subjects. Neurosci Lett 2004;356:228-230.

19. Cuccia A, Caradonna C. The relationship between the stomatognathic system and body posture. Clinics 2009;64:6166.

20. Kirveskari P, Jamsa T. Health risk from occlusal interferences in females. Eur J Orthod 2009;31:490-495.

21. Cao Y, Xie QF, Li K, Light AR, Fu KY. Experimental occlusal interference induces long-term masticatory muscle hyperalgesia in rats. Pain 2009;144:287-293. 